\title{
Dual Fraunhofer interference and charge fluctuations in long quantum phase slip wires
}

\author{
S. E. de Graaf (1)* \\ National Physical Laboratory, Hampton Road, TW11 OLW Teddington, United Kingdom
}

(Received 13 July 2020; accepted 28 September 2020; published 12 October 2020)

\begin{abstract}
Charge interference (Aharonov-Casher effect) in a long superconducting quantum phase slip wire is considered, and from this the "dual" Fraunhofer interference effect (dual to the critical current modulation of a short Josephson junction in an external magnetic field) is derived. The device that can be used to observe this effect is proposed. Furthermore, the impact of wire inhomogeneities, charge disorder, and noise on the phase slip amplitude is investigated. Although intrinsically protected against small fluctuations, the Aharonov-Casher interference resulting from jumps of random offset charges and quasiparticles can result in significant fluctuations of the measured current-voltage characteristics of the quantum phase slip wire, similar to the effects of Joule heating when averaged out over many fluctuations. Possible ways to identify and mitigate such disorder are discussed.
\end{abstract}

DOI: 10.1103/PhysRevB.102.144509

\section{INTRODUCTION}

Significant efforts have been made in the past decades to observe so-called "dual" Shapiro steps, an important milestone towards realizing a quantum current standard, dual to that of the Josephson volt $[1,2]$. Experimental demonstration of robust dual Shapiro steps remains challenging [3,4], and a voltage blockade due to coherent quantum phase slips (CQPS) [5], the dual of Cooper pair tunneling, is often difficult to distinguish from conventional Coulomb blockade due to wire inhomogeneities [6]. Alternate methods of verifying the existence and stability of CQPS in superconducting nanowires would, therefore, aid in the development of devices based on the dual CQPS effect.

The demonstration of Fraunhofer interference in short Josephson junctions [7] was important in verifying the Josephson effect. Likewise, similar interference in the critical voltage of a superconducting nanowire should constitute clear evidence for coherent quantum phase slips [8]. Although simple duality arguments can be used to cast the equations for the classical Fraunhofer interference pattern in CQPS devices [1], a microscopic derivation of this phenomena is still lacking. The Fraunhofer interference in Josephson junctions can be understood in terms of the Aharonov-Bohm effect where a charged particle (Cooper pair) can move along different trajectories around a static fluxon. The dual to this effect is the Aharonov-Casher (AC) effect [9] where fluxon interference, carried by quantum phase slips, is obtained when trajectories encircle static charges.

\footnotetext{
*sdg@npl.co.uk
}

Published by the American Physical Society under the terms of the Creative Commons Attribution 4.0 International license. Further distribution of this work must maintain attribution to the author(s) and the published article's title, journal citation, and DOI.
The AC effect has been considered extensively in systems of Josephson junctions in the phase slip regime (or analogously in nanowires with Josephson coupling between grains) [10-17]. In particular, the effects of disorder both due to background charge (parity) fluctuations [16-23] and spatial variations in junction parameters [15,23-25] has been studied in detail. The AC effect has also been demonstrated experimentally for phase slips in Josephson junction rings [10] and networks [12,26] as well as for CQPS in devices made of continuous superconducting wires [27-29], yet the appearance of the direct dual to the diffraction effects in Josephson junctions is lacking. However, such an effect would form striking evidence for CQPS in transport measurements of superconducting nanowires.

Here, the dual Fraunhofer interference for a long homogeneous phase slip wire is derived, and conditions under which it can be observed are discussed. From this, the role of disorder and noise, due to local charges, on the phase slip rate is derived. Random offset charges and charge fluctuations along the CQPS wire are found to suppress the phase slip rate, obscure the diffraction effects, and result in smearing of measured current-voltage (IV) characteristics with similar appearance as, e.g., overheating effects.

\section{MODEL}

In what follows, a voltage biased superconducting nanowire of length $L$ is considered. The length $L$ is much longer than the charge localization length $\zeta$, and the wire is assumed to be deep in the phase slip regime. The currentvoltage characteristics is given by a critical voltage $V_{c 0}$ (dual to the critical current for a Josephson junction) due to frequent CQPS resulting in a total phase slip rate $\left|v_{\text {tot }}\right|=2 \mathrm{eV} V_{c 0} / \hbar$. Below $V_{c 0}$, no current flows through the device, and above $V_{c 0}$, the nanowire transitions to the resistive state, typically characterized by a large sheet resistance $R_{\mathrm{sq}} \sim R_{q}$, where $R_{q}=h / 4 e^{2}$ is the superconducting resistance quantum. 
(a)

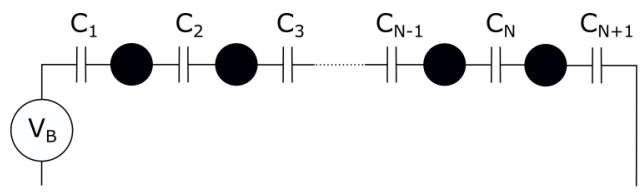

(b)

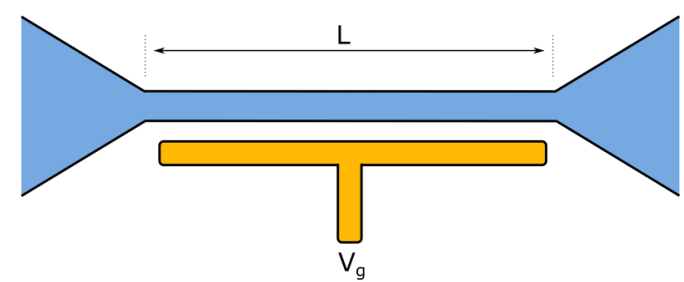

(c)

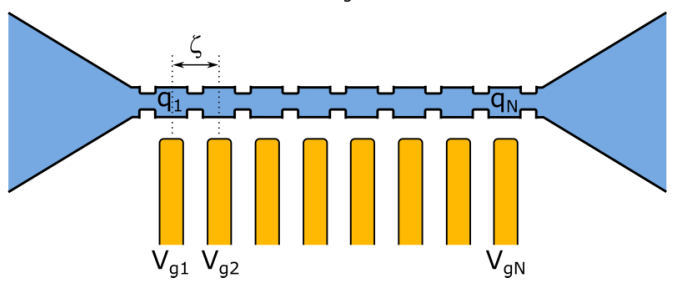

FIG. 1. (a) Circuit model for an array of capacitively coupled islands, in between which frequent CQPS can occur. (b) Proposed CQPS nanowire device for the observation of dual Fraunhofer interference for phase slips. (c) A modified implementation of (b) which allows for compensation of individual offset charges and local control of the phase slip rate using multiple gates separated by a characteristic distance $\zeta$.

To generalize the problem, an approximate picture can be used to provide an intuitive description of the AC effect. In this picture, the CQPS wire consists of a chain of $N$ capacitively coupled islands [Fig. 1(a)], and phase slips (fluxon tunneling) are permitted in between these islands [17]. As it turns out, any Josephson or CQPS coupling between the islands is irrelevant for the physics in the AC picture described here. The junction chain is, in this respect, topologically identical to the homogeneous CQPS wire. A complete microscopic description for a CQPS wire can be derived from the full hydrodynamic action under the sine-Gordon model which assumes the CQPS element to be a zero-dimensional lumped-element object with a constant charge distribution throughout the wire [30,31]. However, under this approximation, the potential impact of induced (local) charges are usually approximated out, and the CQPS action becomes insensitive to local variations in charge. Although inclusion of an AC-like term can be accounted for explicitly [32], an exact solution of the full hydrodynamic CQPS action is, in general, intractible. Here, the bare phase slip rate is assumed to be $a$ priori determined, and only its modification due to the $\mathrm{AC}$ contribution is considered.

To translate the junction chain back onto the CQPS nanowire, one should consider the renormalization of the relevant length scales, mapping the number of islands $N$ onto a characteristic length $\zeta$ in the wire, i.e., $N \sim L / \zeta$. For a highly disordered CQPS wire deep in the phase slip regime with $R_{\mathrm{sq}} \sim R_{q}$, the relevant length scale for charge localization is on the order of 1 square (of size $\zeta \sim w, w$ being the wire width), and if the CQPS nanowire is made up of a granular film with weak couplings between grains, the grain size, instead, becomes the relevant scale. Alternatively, for a homogeneous wire dominated by order parameter disorder $[33,34]$ the BCS coherence length $\xi_{0}$ forms the relevant length scale.

\section{A. Phase slip rate}

To calculate the $\mathrm{AC}$ contribution to the phase slip rate in the circuit depicted in Fig. 1(a), the Matveev-Larkin-Glazman theory $[10,17]$ is used to calculate the phase slip rate $v_{m}=$ $\nu_{0} e^{-S_{m}}$ between each grain from the imaginary time-action $S_{m}$, which yields the total phase slip rate of the long wire as

$$
v_{\text {tot }}=\sum_{m}^{N} v_{0} e^{-S_{m}} \text {. }
$$

Starting with the Hamiltonian for an array of capacitively coupled Josephson junctions, the Josephson coupling itself can be neglected as this term does not contribute to the AC interference effect [17],

$$
H=\frac{1}{2} \sum_{i, j}\left[C^{-1}\right]_{i j}\left(Q_{i}-q_{i}\right)\left(Q_{j}-q_{j}\right) .
$$

Here, $Q_{i}$ is the charge operator and $q_{i}$ is the induced charge (applied via the gate) on each grain, respectively, normalized to $2 e$. Assuming only nearest-neighbor coupling, and equal coupling capacitances $C_{i, i}=2 C, C_{i, \pm 1}=C$ after a transformation to the conjugate phase variable, this results in $H=$ $\frac{1}{2} \sum_{i} C\left(\dot{\theta}_{i}\right)^{2}$, where $\theta_{i}=\varphi_{i+1}-\varphi_{i}$ is the phase difference between islands. The charge contribution to the Lagrangian is, then, given by

$$
\mathcal{L}=\frac{1}{2} \sum_{i} C\left(\dot{\theta}_{i}\right)^{2}-\sum_{i} p_{i} \dot{\theta}_{i},
$$

with $p_{i}=\sum_{k=1}^{i-1} q_{k}$. This sum can be understood in terms of different trajectories of a flux around the island charges, and each trajectory has a different phase shift associated with it. This phase shift is obtained from the total number of encircled charges. I.e., a flux tunneling in the first and $N$ th junction will pick up a phase shift proportional to $\sum_{i=1}^{N} q_{i}$. It can straightforwardly be shown that the first term in Eq. (3) does not contribute to the AC effect [10]. Thus, the imaginary time action for a phase slip event in the $m$ th junction $\delta \theta_{m}=2 \pi$ can be written as

$$
S_{m}=-i \int d t \mathcal{L}_{m}=-2 \pi i p_{m} .
$$

From this, the total charge-dependent phase slip rate of the wire is found to be

$$
v_{\mathrm{tot}}=\sum_{m=1}^{N} v_{0} \eta_{m} \exp \left[i 2 \pi \sum_{k=1}^{m-1}\left(q_{k}+\delta_{q, k}\right)\right] \text {, }
$$

where the two new parameters $\delta_{q, k} \in(-1,1)$ and $\eta_{m} \in(0,1)$ account for charge disorder and disorder in the local phase slip rate, respectively. If all $q_{k}, \delta_{q, k}=0$, and $\eta_{m}=1$, it follows that $v_{\text {tot }}=N v_{0}$.

Importantly, in order to derive Eq. (4), it is assumed that the phases of the left and right leads are the same $\varphi_{R}=\varphi_{L}$ (boundary conditions of a superconducting ring), and, in the event of a phase slip, the phase difference $2 \pi$ appearing between two of the grains is compensated for by adjusting the 
phase difference between all the grains by $-2 \pi / N$ [10]. Since this response is equal for all junctions, it does not contribute to the AC effect. In the case of a voltage-biased circuit, the situation is similar. The phase difference is, instead, pinned by the voltage source such that $\dot{\varphi}_{R}=\dot{\varphi}_{L}-2 e V_{b} / h$, where $V_{b}$ is the bias voltage. Here, on the same grounds, the restoring response to a $2 \pi$ phase slip event in the chain would not contribute to the AC part of the action [22,35].

\section{B. Dual Fraunhofer interference}

In the limit of zero fluctuations, the behavior becomes that of a single island $(N=1)$ connected to reservoirs by two phase slip centers $v_{\text {tot }}=2 e^{\pi i q} \cos (\pi q)$. Such charge modulation of the phase slip rate has been observed in several experiments [13,27-29]. For a long chain of $N$ islands, each having the same charge $q_{i}=q$, it is straightforward to show that Eq. (5) can be written as

$$
v_{\text {tot }}=v_{0} \frac{\sin (N \pi q)}{\sin (\pi q)} e^{\pi i q(N+1)} .
$$

Experiments are insensitive to the exponential phase factor as the measured quantity is $\left|v_{\text {tot }}\right|$. Figure 1 (b) shows a proposed device that can be used to show this effect: A homogeneous superconducting wire in the phase slip regime is placed in the vicinity of a gate electrode that uniformly induces the same charge along the whole wire through a distributed gate capacitance $C_{g}$. The dual Fraunhofer interference is exhibited through the measured critical voltage $V_{c}$ in a transport experiment that varies with applied gate voltage $V_{g}$. Equation (6) can, in the limits $q \ll 1$ and $N \gg 1$, be approximated as

$$
\left|v_{\text {tot }}\right| \approx v_{0}\left|\frac{\sin (N \pi q)}{\pi q}\right|,
$$

which is the more familiar expression for the Fraunhofer interference. This is the CQPS dual of the critical current $I_{c}$ diffraction in short Josephson junctions subject to an applied external magnetic flux $\Phi: I_{c}=I_{c 0}\left|\sin \left(\pi \Phi / \Phi_{0}\right)\right| /\left|\pi \Phi / \Phi_{0}\right|$.

In Fig. 2, Eqs. (6) and (7) are plotted. The insets show the evolution of the phase slip rate on the complex plane at the points indicated. Each individual phase slip center contributes with an additional rotation on the complex plane due to the induced charge on the island, resulting in the increased curvature of the "phase slip rate contour" of fixed length. The phase slip rate, thus, experiences minima when the accumulated island charge is a multiple of $2 e$. For each such period, the diameter of the circle on the complex plane shrinks due to multiple windings, which results in the overall reduction in the phase slip rate.

The full interference pattern [Eq. (6)] is also $2 e$ periodic in the charge of a single island. This is an interesting phenomena which does not have an analog for the critical current modulation of a short Josephson junction, arising from the fundamentally different nature of the interfering particles. Note also that a classical Coulomb blockade system would maintain the same Coulomb gap (critical voltage) over many periods in gate charge, whereas the critical voltage due to CQPS in long wires must be quickly suppressed. As such, the observation of a dual Fraunhofer pattern would constitute

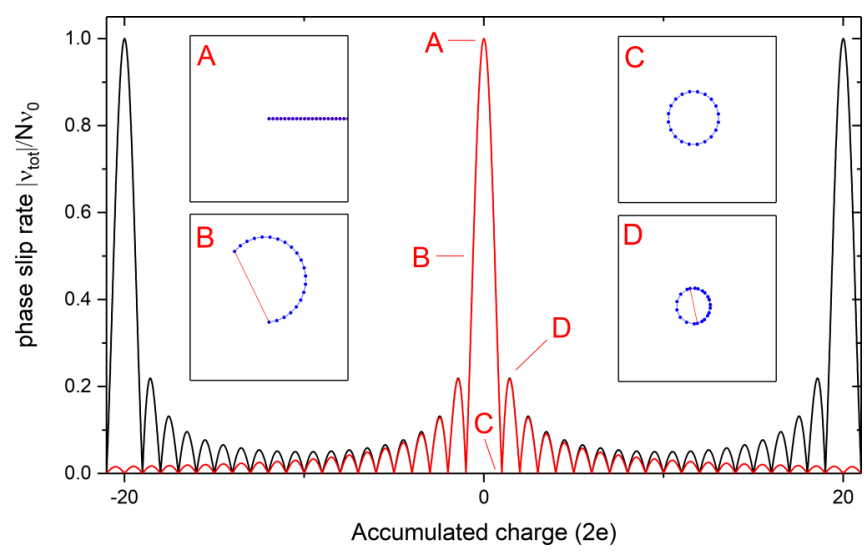

FIG. 2. Phase slip rate for a homogeneous QPS wire without charge disorder as a function of accumulated induced charge along the wire $(N=20)$ according to Eq. (6) (solid black line). The approximate expression Eq. (7) is shown as the red solid line. The insets show the evolution of the phase slip rate in the complex plane where the total arc length is always the same $\left(=N v_{0}\right)$, and the accumulated charge along the wire results in a phase factor for each individual phase slip center.

unambiguous evidence for CQPS in a DC experiment on long superconducting wires.

However, in reality, this dual effect may be challenging to observe: It requires a long and homogeneous CQPS wire free of offset charges and with a uniformly applied gate voltage. For increased control over these effects, the device sketched in Fig. 1(c) is proposed. A large number of individual gate electrodes can be used to equilibrate any offset charges, and locations for phase slips are well defined by the use of local constrictions [25]. Alternatively, controlled locations for phase slips can be achieved by locally modifying the superconductor properties [36]. A similar multi-CQPS junction geometry has been proposed for a CQPS qubit device [37] (using the assumed dual Hamiltonian).

\section{Offset charges}

The above discussion is in the limit of no charge disorder, however, a more likely scenario is that each island is subject to a random offset charge. These offset charges could, for instance, be due to local charged material defects or quasiparticles. The general effect of such offset charges would be a reduction of the total measured phase slip rate $\left|v_{\text {tot }}\right|<N\left|v_{0}\right|$. For large random offset charges, the phase slip rate can be interpreted as a random walk on the complex plane which results in $v_{\text {tot }}=v_{0} \sqrt{N}$ [17]. Although such a description gives an appropriate estimate for the expectation value for the phase slip rate, the offset charges are seldom static. In fact, they typically fluctuate on timescales commensurate with experimental durations, resulting in variations in the measured phase slip rate.

There are two regimes of such fluctuations. (i) Strong (slow stochastically switching) variations $\delta q_{i} \sim 1$ due to local strongly coupled charge fluctuators or quasiparticles, and (ii) weak (and typically high-frequency) fluctuations characterized by $\delta q_{i} \ll 1$, originating from fluctuations in the wider charge environment. Case (i) is discussed in what follows, and case (ii) is discussed in the next section. 

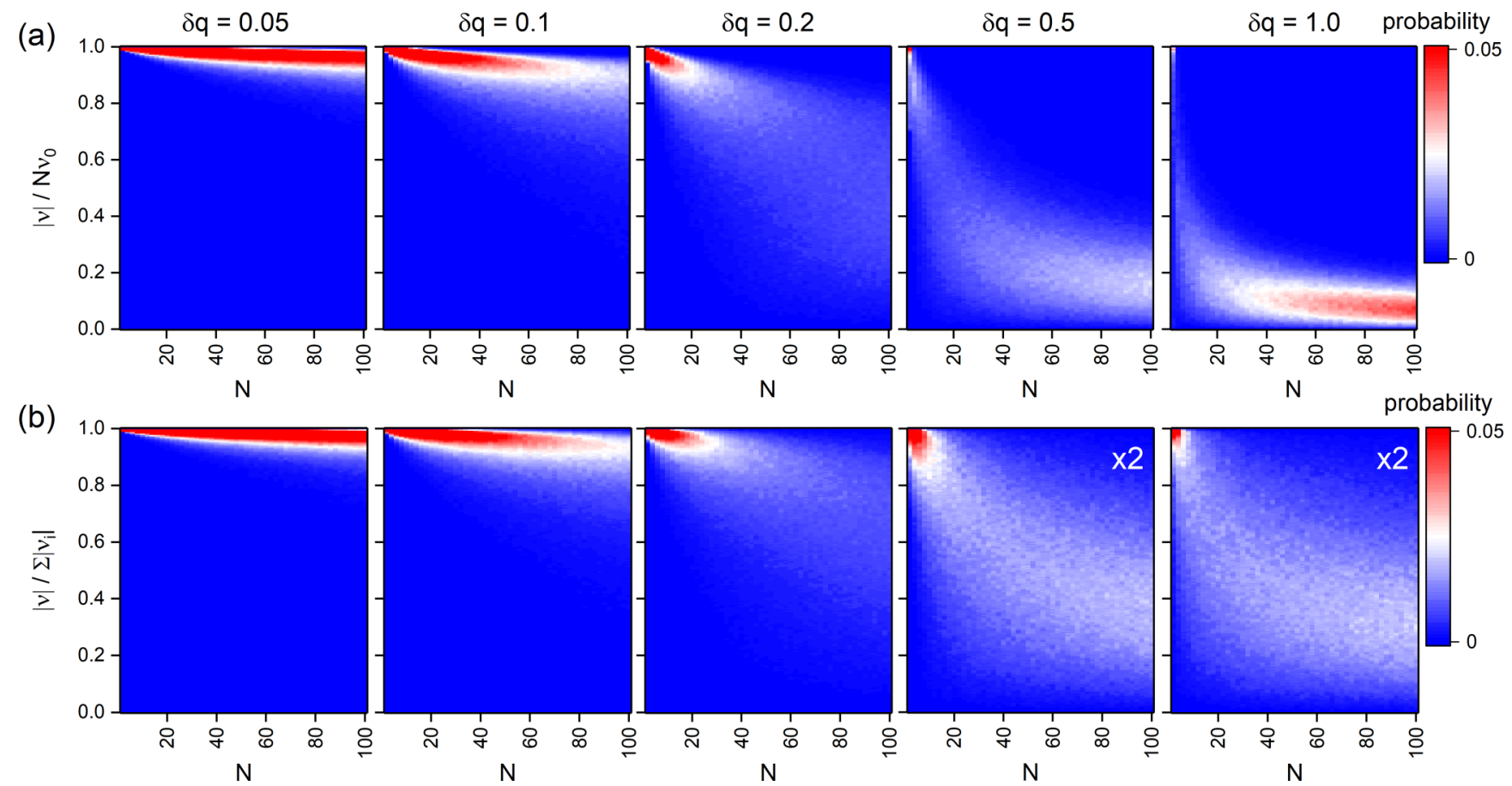

FIG. 3. (a) Probability distribution for the total phase slip rate $|v| / N v_{0}$ for increasing magnitude of the random charge disorder along the nanowire $\delta q(2 e)$. b) The same probability distributions as in (a) but with added disorder in the nanowire width. Here, the normalization in the phase slip rate is taken as the sum of individual random phase slip rates $|v| / \sum_{i}\left|v_{i}\right|$. The calculation assumes a wire with normal state per square resistance $0.3 R_{q}, \xi_{0}=4 \mathrm{~nm}$ and a mean wire width $w=20 \mathrm{~nm}$ with a normal distributed dispersion of width $\delta w=5 \mathrm{~nm}$. Note that in the last two panels the probability has been multipled by a factor of 2 for enhanced visibility.

Figure 3(a) shows the probability distribution of obtaining a given phase slip rate $\left|v_{\text {tot }}\right|$ as a function of $N$ for a uniform random distribution of static offset charges on the interval $(-\delta q, \delta q)$ for selected values of $\delta q$. For small $\delta q,\left|v_{\text {tot }}\right| \approx$ $N\left|v_{0}\right|$. As $\delta q$ approaches 1 , the probability for a reduced phase slip rate increases substantially for larger $N$, and the width of the distribution is also significantly widened. For $\delta q \rightarrow 1$, the expected $\left|v_{\text {tot }}\right| \rightarrow \sqrt{N}\left|v_{0}\right|$ dependence is recovered with a suppressed distribution width.

A realistic QPS nanowire does not only suffer from local offset charges, but also local variations in the phase slip rate due to a dispersion in wire properties will result in additional disorder. In particular, the exponential dependence of the phase slip amplitude on the wire width [38] will have a significant impact. Such dispersion in wire width is modeled in Fig. 3(b) by assuming a normal distribution of width $\delta w=$ $5 \mathrm{~nm}$ and mean $w=20 \mathrm{~nm}$ and considering typical parameters for $\mathrm{NbN}$ [38]: $v_{0}=v_{0}^{\prime} \exp (-\kappa w)$ with $\kappa=a R_{q} / R_{\mathrm{sq}} \xi_{0}=$ $2 \mathrm{~nm}^{-1}$ and $a=0.6$. Such dispersion in the nanowire width results in a recovery of a larger phase slip rate for large $N$. It also results in significant broadening of the probability distribution as the total phase slip rate is governed by a fewer number of strong phase slip centers. As the random offset charges discussed above [and Fig. 3(a)] can only be considered static for a given instance in time (these strong offset charges could also fluctuate in time) the result is that $v_{\text {tot }}$ can fluctuate in time within a range of values given by the strength of the disorder [Figs. 3(a) and 3(b)]. As shown below, this can result in significant smearing of the current-voltage characteristics of a CQPS wire.

\section{Charge noise}

Next, the impact of small fluctuations $\left(\delta q_{i} \ll 1\right)$ is studied with the aim to calculate the noise power spectral density of the phase slip rate as a function of the charge noise power spectral density along the wire. Here, it is assumed that the charge noise between individual islands is uncorrelated. The spectral density of fluctuations in the phase slip rate is defined as

$$
S_{\nu}(\omega)=\frac{1}{2 \pi} \int d t \int d \tau\langle v(t), v(t+\tau)\rangle e^{-2 \pi i \omega \tau},
$$

where $\langle$,$\rangle denotes covariance. Inserting the expression for the$ phase slip rate into Eq. (8) gives

$$
S_{\nu}(\omega)=\frac{\nu_{0}^{2}}{2 \pi} \int d t \int d \tau \sum_{k=0}^{N} \sum_{m=0}^{N}\left\langle e^{P_{k}+p_{k}(t)}, e^{P_{m}+p_{m}(t+\tau)}\right\rangle e^{-2 \pi i \omega \tau},
$$

Here, the charge on each island is split into a static part $P_{k}=2 \pi i \sum_{n=1}^{k} Q_{k}$ and a time-dependent part with zero mean $p_{k}(t)=2 \pi i \sum_{n=1}^{k} q_{k}(t)$. Next, it is assumed that $\left\langle q_{l}(t), q_{m}(t+\tau)\right\rangle=0$ for all $l \neq m$. This assumption is justified by the fact that, whereas neighboring islands may still have correlations in their time-dependent charge fluctuations, a length scale at which fluctuations become uncorrelated can be defined. If such length scales span over several islands, the effective number of islands $N$ is reduced. Furthermore, it is assumed that all $q_{m}$ obey the same statistics: $\left\langle q_{m}(t), q_{m}(t+\right.$ $\tau)\rangle \equiv\langle q(t), q(t+\tau)\rangle$. This means that

$$
\left\langle p_{l}(t), p_{m}(t+\tau)\right\rangle=2 \pi i \min (l, m)\langle q(t), q(t+\tau)\rangle .
$$


Finally, since $P_{k}$ is constant with respect to $t$, this becomes

$$
S_{\nu}(\omega)=\frac{v_{0}^{2}}{2 \pi} \int d t \int d \tau \sum_{k=0}^{N} \sum_{m=0}^{N} e^{P_{k}} e^{P_{m}}\left\langle e^{p_{k}(t)}, e^{p_{m}(t+\tau)}\right\rangle e^{-2 \pi i \omega \tau}
$$

Now, assuming that the charge fluctuations are small, i.e., expanding the exponents to first order, leads to

$$
S_{\nu}(\omega)=2 \pi S_{q}(\omega) i v_{0}^{2} \sum_{k=0}^{N} \sum_{m=0}^{N} e^{P_{k}+P_{m}} \min (k, m),
$$

where

$$
S_{q}(\omega)=\frac{1}{2 \pi} \int d t \int d \tau\langle q(t), q(t+\tau)\rangle e^{-2 \pi i \omega \tau}
$$

is the charge noise power spectral density. Without static charge disorder, the sum in Eq. (11) becomes $\sum_{k=0}^{N} \sum_{m=0}^{N} \min (k, m)=\frac{N}{6}+\frac{N^{2}}{2}+\frac{N^{3}}{3}$. For the ideal long wire, when all $P_{k}=n 2 \pi$, the absolute noise power in the phase slip rate will scale as $S_{v}(\omega) \approx 2 \pi i S_{q}(\omega) v_{0}^{2} N^{3} / 3$, however, this fluctuation is orthogonal to the static phase slip amplitude. This means that the measured amplitude fluctuations of the phase slip rate scales as $\propto N$ for large $N$ in the absence of static charge disorder, a result verified by Monte Carlo simulations of Eq. (9).

On the contrary, for large random static offset charges, the impact of the local charge noise $\left(\delta q_{i} \ll 1\right)$ is reduced. In fact, $S_{v}(\omega) \propto S_{q}(\omega)$ can be found numerically, showing no dependence on $N$. This is an important result, highlighting that the ideal CQPS wire (free of large offset charges) is to first order protected from low amplitude charge noise since this Aharonov-Casher-type noise results in fluctuations that are orthogonal to the measured phase slip rate on the complex plane. With large charge disorder, this protection towards small fluctuations is enhanced. Instead, the dominant source of fluctuations impacting device performance will come from slow switching of the much stronger random charge disorder of magnitude $\delta q \sim 1 e$ along the wire.

\section{CURRENT-VOLTAGE CHARACTERISTICS}

Numerical calculations of the expected IV characteristics can be performed to understand the impact of strong charge fluctuations. Here, the CQPS wire is described by its critical voltage $V_{c}(t)=2 \pi E_{s}(t) / 2 e$, where $E_{s}(t)=h\left|v_{\text {tot }}(t)\right|$ is the phase slip energy. For a voltage-biased QPS junction in series with an inductor $L$ and a resistor $R$ leading to the equation of motion [1],

$$
L \ddot{q}+2 e R \dot{q}+V_{c}(t) \sin (2 \pi q)=V_{b}+V_{\mathrm{rf}} \sin \omega_{\mathrm{rf}} t .
$$

This charge representation allows for both QPS and the boundary conditions for the phase arising from the environment, which are now reduced to Kirchhoff's equation for the charge to be well defined. Using Monte Carlo simulations where $V_{c}(t)$ varies according to a normal distribution of mean $V_{c 0}=33 \mu \mathrm{V}$ and width $\sigma_{q}=0.1 V_{c 0}$, which represents a modest spread for a long disordered wire [cf. Fig. 3(b)]. The simulations evaluate the resulting average current $I \propto \dot{q}$ as a function of $V_{b}$, in the presence of a microwave drive with

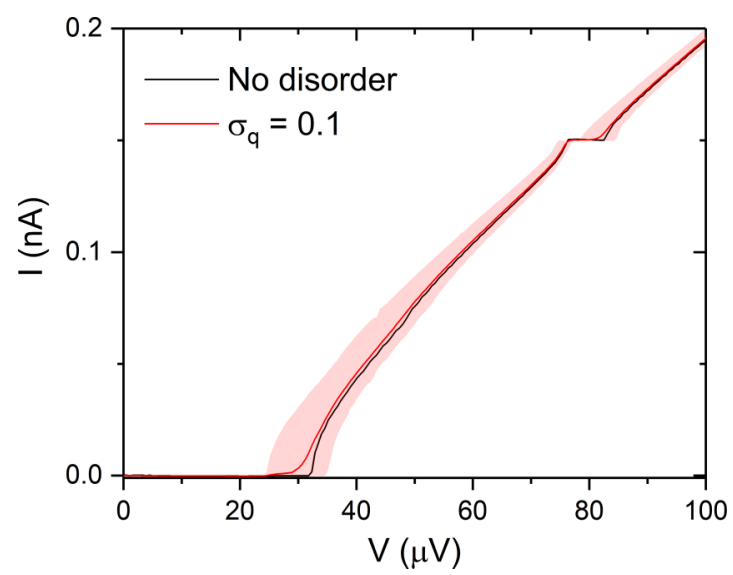

FIG. 4. Simulated current voltage characteristics of a long CQPS wire with fluctuating charge disorder under the presence of microwave radiation at $\omega_{\mathrm{rf}} / 2 \pi=0.5 \mathrm{GHz}$. The black solid line shows the case without disorder, and the solid red line shows the average of 20 different charge configurations with a moderate total phase slip rate dispersion of $\sigma_{v}=0.1 E_{s 0}$. The shaded region indicates the possible spread of parameters for the given probability distribution of phase slip rates. $E_{L}=e \Phi_{0}^{2} / L=0.25, E_{s 0}=2.5 \mathrm{GHz}, R=$ $320 \mathrm{k} \Omega$.

angular frequency $\omega_{\mathrm{rf}}$ and amplitude $V_{\mathrm{rf}}$ that generates a dual Shapiro step at $I=2 e \omega_{\mathrm{rf}} / 2 \pi$.

The results are shown in Fig. 4, which evaluate the effect of charge fluctuations on the transport characteristics of the phase slip wire. Figure 4 shows that the smearing of the IV curve due to averaging over a number of charge fluctuations looks qualitatively very similar to other effects such as heating [39] or insufficient filtering. Crucially, whereas the zero bias blockade is still visible, plateaus due to applied microwave radiation are more easily washed out. Therefore, these simulations conclude that charge fluctuations in long quantum phase slip wires is a major issue towards the observation of robust dual Shapiro steps, in particular, due to the small voltages and currents used that usually require long averaging times.

The $1 / f$ flicker critical voltage noise inherited from the charge noise discussed, here, can be considered dual to the critical current noise found in Josephson junctions and superconducting quantum interference devices where bias reversal and modulation techniques have successfully been applied to reduce noise and elude its origin [40,41]. Applying similar techniques in experiments on CQPS wires and exploiting the fact that the $1 / f$ critical voltage noise would be sensitive to (local) gate voltage, could distinguish it from white Johnson [39] or shot noise [42] effects, that are also expected to have a different temperature dependence. To achieve this, high bandwidth low-noise (pulsed) readout for CQPS wires is required.

\section{DISCUSSION}

A number of factors can contribute to the charge and phase slip amplitude disorder in superconducting nanowires. So far, the result of such variations on the total phase slip rate have been considered without any assumption of the underlying mechanisms. 
The presence of charge disorder is a long-standing problem for materials for microscopic quantum circuits [43], and the most successful devices developed have been engineered to, instead, be insensitive to charge and parity fluctuations. A different approach has been taken by the two-dimensional (2D) materials community where materials engineering and encapsulation have resulted in suppressed charge disorder and significantly improved device performance [44]. Exploiting such techniques in conjunction with superconductivity in novel 2D materials $[45,46]$ may provide an alternative route for CQPS which potentially could solve some of the challenges with spatial inhomogeneities as well. Variations in wire cross sections can easily emerge as a result of imperfect lithography defining the nanowires as well as from uncontrolled oxidation of the superconducting material or thickness variations. A similar dispersion in phase slip rate can originate from both intrinsic fluctuations of the order parameter close to the superconductor-insulator transition [33] or due to external magnetic moments and defects locally modifying the order parameter [47]. Nonequilibrium quasiparticles will also contribute to fluctuations in the phase slip rate. Such quasiparticles are notoriously challenging to eliminate [48], are expected to be present in larger numbers in highly disordered superconductors otherwise suitable for CQPS, and they could also become trapped in a spatially fluctuating order parameter and contribute to the local charge disorder [49]. The inclusion of local electrostatic gating for CQPS nanowires [6] provides a separate degree of freedom that can help in further understanding CQPS physics.

\section{SUMMARY}

Here, the CQPS dual of the well-known Fraunhofer interference in short Josephson junctions has been derived, and it is shown how this interference arises from the AharonovCasher effect in long CQPS wires. A device is proposed that can be used to demonstrate this effect. A major challenge towards its experimental observation is charge noise, random offset charges, and inhomogeneities along the CQPS wire. The charge noise spectral density was shown to enter into the fluctuations of the phase slip rate, and how the phase slip rate is affected by local disorder was investigated. Rather surprisingly, high-frequency charge noise is suppressed in a long CQPS wire, however, significant fluctuations in the phase slip rate is still expected due to slow random offset charge jumps of magnitude $\sim 1 e$. The Josephson junction analogy is a reduced critical current of the junction in the presence of a magnetic field with the important difference that electric fields due to stray charge are a local effect. When multiple charge jumps are averaged over experimental timescales, the measured current voltage characteristics of a CQPS wire shows smearing which is similar to that of, e.g., joule heating. These results show that it is important to control and understand the local charge environment of CQPS wires in order to develop practical applications based on dual superconducting circuits.

\section{ACKNOWLEDGMENTS}

The author is grateful to D. S. Golubev, A. Y. Tzalenchuk, and A. Kemppinen for useful discussions and J. J. Burnett and T. Lindström for careful reading of the paper. This work has received funding from the European Union's Horizon 2020 Research and Innovation Programme under Grant Agreement No. 862660/QUANTUM E-LEAPS and the UK government's Department for Business, Energy and Industrial Strategy through the UK National Quantum Technologies Programme.
[1] J. E. Mooij and Y. V. Nazarov, Nat. Phys. 2, 169 (2006).

[2] W. Poirier, S. Djordjevic, F. Schopfer, and O. Thévenot, C. R. Phys. 20, 92 (2019).

[3] J. S. Lehtinen, K. Zakharov, and K. Y. Arutyunov, Phys. Rev. Lett. 109, 187001 (2012).

[4] Z. M. Wang, J. S. Lehtinen, and K. Y. Arutyunov, Appl. Phys. Lett. 114, 242601 (2019).

[5] O. V. Astafiev, L. B. Ioffe, S. Kafanov, Y. A. Pashkin, K. Y. Arutyunov, D. Shahar, O. Cohen, and J. S. Tsai, Nature (London) 484, 355 (2012).

[6] S. E. de Graaf, R. Shaikhaidarov, T. Lindström, A. Y. Tzalenchuk, and O. V. Astafiev, Phys. Rev. B 99, 205115 (2019).

[7] J. M. Rowell, Phys. Rev. Lett. 11, 200 (1963).

[8] A. J. Kerman, New J. Phys. 15, 105017 (2013).

[9] Y. Aharonov and A. Casher, Phys. Rev. Lett. 53, 319 (1984).

[10] I. M. Pop, B. Doucot, L. Ioffe, I. Protopopov, F. Lecocq, I. Matei, O. Buisson, and W. Guichard, Phys. Rev. B 85, 094503 (2012).

[11] J. R. Friedman and D. V. Averin, Phys. Rev. Lett. 88, 050403 (2002).
[12] W. J. Elion, J. J. Wachters, L. L. Sohn, and J. E. Mooij, Phys. Rev. Lett. 71, 2311 (1993).

[13] M. T. Bell, W. Zhang, L. B. Ioffe, and M. E. Gershenson, Phys. Rev. Lett. 116, 107002 (2016).

[14] R. Süsstrunk, I. Garate, and L. I. Glazman, Phys. Rev. B 88, 060506(R) (2013).

[15] F. W. J. Hekking and L. I. Glazman, Phys. Rev. B 55, 6551 (1997).

[16] D. A. Ivanov, L. B. Ioffe, V. B. Geshkenbein, and G. Blatter, Phys. Rev. B 65, 024509 (2001).

[17] K. A. Matveev, A. I. Larkin, and L. I. Glazman, Phys. Rev. Lett. 89, 096802 (2002).

[18] J. H. Cole, A. Heimes, T. Duty, and M. Marthaler, Phys. Rev. B 91, 184505 (2015).

[19] S. Khlebnikov and L. P. Pryadko, Phys. Rev. Lett. 95, 107007 (2005).

[20] K. Cedergren, R. Ackroyd, S. Kafanov, N. Vogt, A. Shnirman, and T. Duty, Phys. Rev. Lett. 119, 167701 (2017).

[21] M. Bard, I. V. Protopopov, I. V. Gornyi, A. Shnirman, and A. D. Mirlin, Phys. Rev. B 96, 064514 (2017).

[22] H.-K. Wu, and J. D. Sau, Phys. Rev. B 99, 214509 (2019). 
[23] A. E. Svetogorov, M. Taguchi, Y. Tokura, D. M. Basko, and F. W. J. Hekking, Phys. Rev. B 97, 104514 (2018).

[24] A. E. Svetogorov and D. M. Basko, Phys. Rev. B 98, 054513 (2018).

[25] M. Vanević and Y. V. Nazarov, Phys. Rev. Lett. 108, 187002 (2012).

[26] A. Roy, Y. Wu, R. Berkovits, and A. Frydman, Phys. Rev. Lett. 125, 147002 (2020).

[27] S. E. de Graaf, S. T. Skacel, T. Hönigl-Decrinis, R. Shaikhaidarov, H. Rotzinger, S. Linzen, M. Ziegler, U. Hübner, H.-G. Meyer, V. Antonov et al., Nat. Phys. 14, 590 (2018).

[28] S. Kafanov and N. M. Chtchelkatchev, J. Appl. Phys. 114, 073907 (2013).

[29] T. T. Hongisto and A. B. Zorin, Phys. Rev. Lett. 108, 097001 (2012).

[30] S. Khlebnikov, Phys. Rev. B 78, 014512 (2008).

[31] K. Y. Arutyunov, D. S. Golubev, and A. D. Zaikin, Phys. Rep. 464, 1 (2008).

[32] A. Andersson and J. Lidmar, Phys. Rev. B 91, 134504 (2015).

[33] B. Sacépé, C. Chapelier, T. I. Baturina, V. M. Vinokur, M. R. Baklanov, and M. Sanquer, Phys. Rev. Lett. 101, 157006 (2008).

[34] C. Carbillet, V. Cherkez, M. A. Skvortsov, M. V. Feigel'man, F. Debontridder, L. B. Ioffe, V. S. Stolyarov, K. Ilin, M. Siegel, C. Noüs et al., Phys. Rev. B 102, 024504 (2020).

[35] A. M. Hriscu and Y. V. Nazarov, Phys. Rev. B 83, 174511 (2011).

[36] E. Mykkänen, A. Bera, J. S. Lehtinen, A. Ronzani, K. Kohopää, T. Hönigl-Decrinis, R. Shaikhaidarov, S. E. de Graaf, J. Govenius, and M. Prunnila, Nanomaterials 10, 950 (2020).

[37] Z.-Z. Li, T.-F. Li, C.-H. Lam, and J. Q. You, Phys. Rev. A 99, 012309 (2019).
[38] J. T. Peltonen, O. V. Astafiev, Y. P. Korneeva, B. M. Voronov, A. A. Korneev, I. M. Charaev, A. V. Semenov, G. N. Golt'sman, L. B. Ioffe, T. M. Klapwijk, and J. S. Tsai, Phys. Rev. B 88, 220506(R) (2013).

[39] C. H. Webster, J. C. Fenton, T. T. Hongisto, S. P. Giblin, A. B. Zorin, and P. A. Warburton, Phys. Rev. B 87, 144510 (2013).

[40] V. Foglietti, W. J. Gallagher, M. B. Ketchen, A. W. Kleinsasser, R. H. Koch, S. I. Raider, and R. L. Sandstrom, Appl. Phys. Lett. 49, 1393 (1986).

[41] M. Mück, M. Korn, C. G. A. Mugford, J. B. Kycia, and J. Clarke, Appl. Phys. Lett. 86, 012510 (2005).

[42] A. G. Semenov and A. D. Zaikin, Phys. Rev. B 94, 014512 (2016).

[43] E. Paladino, Y. M. Galperin, G. Falci, and B. L. Altshuler, Rev. Mod. Phys. 86, 361 (2014).

[44] M. Yankowitz, Q. Ma, P. Jarillo-Herrero, and B. J. LeRoy, Nat. Rev. Phys. 1, 112 (2019).

[45] Y. Cao, V. Fatemi, S. Fang, K. Watanabe, T. Taniguchi, E. Kaxiras, and P. Jarillo-Herrero, Nature (London) 556, 43 (2018).

[46] N. Paradiso, A.-T. Nguyen, K. Enzo Kloss, and C. Strunk, 2D Mater. 6, 025039 (2019).

[47] N. A. Saveskul, N. A. Titova, E. M. Baeva, A. V. Semenov, A. V. Lubenchenko, S. Saha, H. Reddy, S. I. Bogdanov, E. E. Marinero, V. M. Shalaev et al., Phys. Rev. Appl. 12, 054001 (2019).

[48] A. Vepsäläinen, A. H. Karamlou, J. L. Orrell, A. S. Dogra, B. Loer, F. Vasconcelos, D. K. Kim, A. J. Melville, B. M. Niedzielski, J. L. Yoder et al., Nature (London) 584, 551 (2020).

[49] S. E. de Graaf, L. Faoro, L. B. Ioffe, S. Mahashabde, J. J. Burnett, T. Lindström, S. E. Kubatkin, A. V. Danilov, and A. Y. Tzalenchuk, arXiv:2004.02485. 